\title{
The Arcuate Nucleus of the C57BL/6J Mouse Hindbrain Is a Displaced Part of the Inferior Olive
}

\author{
Yu Hong Fu ${ }^{a}$ Charles Watson ${ }^{a, b}$ \\ ${ }^{a}$ Neuroscience Research Australia, Randwick, N.S.W, and b Faculty of Health Sciences, Curtin University, \\ Perth, W.A., Australia
}

\section{Key Words}

Arcuate nucleus of the hindbrain • Inferior olive .

Precerebellar nucleus

\begin{abstract}
The arcuate nucleus is a prominent cell group in the human hindbrain, characterized by its position on the pial surface of the pyramid. It is considered to be a precerebellar nucleus and has been implicated in the pathology of several disorders of respiration. An arcuate nucleus has not been convincingly demonstrated in other mammals, but we have found a similarly positioned nucleus in the C57BL/6J mouse. The mouse arcuate nucleus consists of a variable group of neurons lying on the pial surface of the pyramid. The nucleus is continuous with the ventrolateral part of the principal nucleus of the inferior olive and both groups are calbindin positive. At first we thought that this mouse nucleus was homologous with the human arcuate nucleus, but we have discovered that the neurons of the human nucleus are calbindin negative, and are therefore not olivary in nature. We have compared the mouse arcuate neurons with those of the inferior olive in terms of molecular markers and cerebellar projection. The neurons of the arcuate nucleus and of the inferior olive share three major characteristics: they both contain neurons utilizing glutamate, serotonin or acetylcholine as neurotransmitters; they both project to the contralateral cer-
\end{abstract}

ebellum, and they both express a number of genes not present in the major mossy fiber issuing precerebellar nuclei. Most importantly, both cell groups express calbindin in an area of the ventral hindbrain almost completely devoid of calbindin-positive cells. We conclude that the neurons of the hindbrain mouse arcuate nucleus are a displaced part of the inferior olive, possibly separated by the caudal growth of the pyramidal tract during development. The arcuate nucleus reported in the C57BL/6J mouse can therefore be regarded as a subgroup of the rostral inferior olive, closely allied with the ventral tier of the principal nucleus.

Copyright $\odot 2012$ S. Karger AG, Basel

\section{Introduction}

The human arcuate nucleus is a group of neurons lying on the ventral aspect of the pyramid of the hindbrain [Mikhail and Ahmed, 1975]. Watson and Paxinos [2010] noted the presence of a similarly positioned nucleus in a calbindin-stained section in their chemoarchitectonic atlas of the mouse brain, and the presence of the nucleus was noted in a study of mouse precerebellar nuclei [Fu et al., 2011]. However, we have found no published account of the characteristics of this nucleus in mice.

Folgering et al. [1979] suggested that the human arcuate nucleus might play a role in central chemoreception,

\section{KARGER}

Fax +41613061234

E-Mail karger@karger.ch

www.karger.com
(C) 2012 S. Karger AG, Basel

0006-8977/12/0793-0191\$38.00/0

Accessible online at:

www.karger.com/bbe
Dr. Charles Watson

Curtin University

Shenton Park Health Research Campus

GPO Box U1987, Perth, WA 6845 (Australia)

Tel. +61 892661 640, E-Mail c.watson@curtin.edu.au 
and other studies have found that this region of the ventral hindbrain in humans is involved in the respiratory response to carbon dioxide concentration, $\mathrm{pH}$ and blood pressure changes [Filiano et al., 1990; Natti, 1991]. Functional magnetic resonance imaging revealed patterns of activation in the arcuate nucleus during hypercapnic ventilatory challenge [Gozal et al., 1994], further suggesting that the arcuate nucleus is involved in respiratory regulation.

Underdevelopment of the arcuate nucleus was reported in a subset of putative human developmental disorders of ventilatory function [Zec et al., 1997]. More recently, abnormalities of the arcuate nucleus have been found in cases of late fetal death [Matturri et al., 2002; Folkerth et al., 2008], in respiratory failure due to multiple system atrophy [Benarroch, 2007], congenital central hypoventilation syndrome [Tomycz et al., 2010] and sudden infant death syndrome [Filiano and Kinney, 1992; Kinney et al., 1995; Paterson et al., 2006b]. These observations provide further evidence that the arcuate nucleus is a primary central chemosensitive area, with a role in the control of automatic breathing in response to hypercapnia. Although some anatomical and pathological studies have been conducted on the human arcuate nucleus, these aspects have not been investigated in mice. The human arcuate nucleus has been historically considered to be a precerebellar nucleus [Brodal, 1981; Zec et al., 1997], but this has not been confirmed by experimental studies.

We undertook a systematic study of the development, connections, cytoarchitecture and chemoarchitectural features of the rodent arcuate nucleus in the belief that it might assist in the understanding of disorders of the human arcuate nucleus. We found that the arcuate nucleus in the mouse is indeed a precerebellar nucleus, and shares calbindin positivity with the inferior olive. However, since the completion of this study, we have learned that the human arcuate nucleus is calbindin negative [J. Baizer, pers. comm.], and is almost certainly a pontine nucleus homolog, and is not in any way homologous with the mouse arcuate nucleus. There is a strong argument for renaming the mouse arcuate nucleus as the prepyramidal subnucleus of the inferior olive.

\section{Animals and Methods}

Animals

C57BL/6J mice (8-10 weeks, 21-25 g, $\mathrm{n}=27$ ) obtained from the Animal Resources Centre (Perth, W.A., Australia), were used for cerebellar injections and histological preparations. Animal experimental procedures were in accordance with the Australian

Code for the Care and Use of Animals in Research, and the procedures were approved by the Animal Care and Ethics Committee of the University of New South Wales.

\section{Surgery and Retrograde Tracing}

Three experiments were performed to map the projections of the arcuate nucleus neurons. Before surgery, all the mice were anesthetized with ketamine $(80 \mathrm{mg} / \mathrm{kg})$ and xylazine $(5 \mathrm{mg} / \mathrm{kg})$ given by intraperitoneal injection. The occipital bone and the atlas on the right side were exposed by retracting the suboccipital muscles.

Experiment 1. To confirm that the arcuate nucleus is one of the precerebellar nuclei, we injected 5\% horseradish peroxidase (HRP; Sigma), into all major regions of the right side of the cerebellum - the anterior vermis, posterior vermis, simple lobule, crus 1 and crus 2 of the ansiform lobule (Crus1 and Crus2), paramedian lobule, paraflocculus and flocculus (fig. 1a; $n=4$ ). HRP is a retrograde tracer readily taken up by fibers terminating in or

\section{Abbreviations used in figures 1-7}

$1 \mathrm{Cb}$

$2 \mathrm{Cb}$

$3 \mathrm{Cb}$

$4 / 5 \mathrm{Cb}$

$6 \mathrm{Cb}$

$7 \mathrm{Cb}$

$8 \mathrm{Cb}$

$10 \mathrm{Cb}$

Ar

ArL

ArM

Crus1

Crus2

FG

Fl

HRP

IC

IO

IOB

IOBe

IOC

IOD

IODM

IOK

IOM

IOPr

IOPrD

IOPrV

IOVL

$\mathrm{PFl}$

PM

py

pye

$\mathrm{RPa}$

Sim lobule 1 of the cerebellar vermis lobule 2 of the cerebellar vermis lobule 3 of the cerebellar vermis lobules 4 and 5 of the cerebellar vermis lobule 6 of the cerebellar vermis lobule 7 of the cerebellar vermis lobule 8 of the cerebellar vermis lobule 10 of the cerebellar vermis arcuate nucleus arcuate nucleus lateral group arcuate nucleus medial group crus 1 of the ansiform lobule crus 2 of the ansiform lobule fluorogold

flocculus

\section{horseradish peroxidase}

inferior colliculus

inferior olive

inferior olive subnucleus B of medial nucleus inferior olive beta subnucleus inferior olive subnucleus $\mathrm{C}$ of medial nucleus inferior olive dorsal nucleus inferior olive dorsomedial nucleus inferior olive cap of Kooy of the medial nucleus inferior olive medial nucleus inferior olive principal nucleus inferior olive principal nucleus dorsal tier inferior olive principal nucleus ventral tier inferior olive ventrolateral protrusion paraflocculus paramedian lobule pyramidal tract pyramidal eminence raphe pallidus nucleus simple lobule 


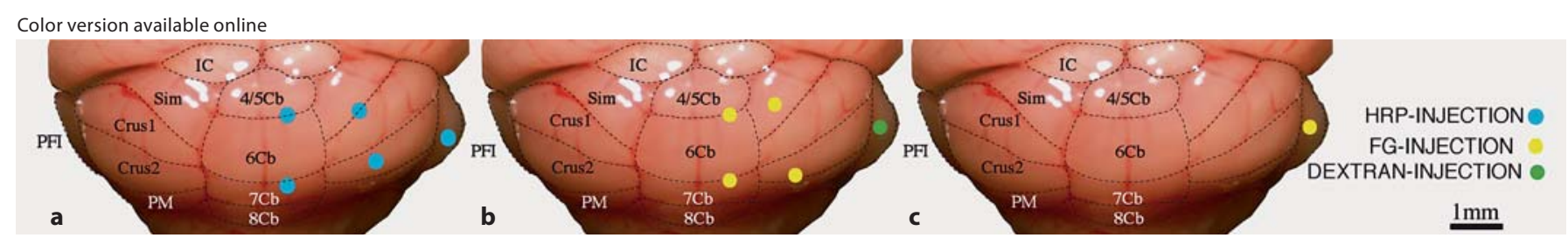

Fig. 1. The location of retrograde tracer injections into the mouse cerebellum. Injections in experiment 1 using HRP (a), experiment 2 using FG and/or AFD injections (b), and experiment 3 using AFD injection (c).

passing through the injection site [Ahlsen, 1981], ensuring the widest anatomical coverage within the hemicerebellum.

Experiment 2. There is evidence of a specific projection from the ventrolateral part of the inferior olive to the paraflocculus of the cerebellum in mammals [Brodal, 1981; Nagao et al., 1997]. Since we believe that this part of the inferior olive gives origin to the arcuate nucleus, we examined its relationship to the paraflocculus. We injected the retrograde tracer Alexa Fluor dextran conjugate (AFD; Alexa Fluor ${ }^{\circledR} 488$; 10,000 MW, No. D-22910, Invitrogen, Carlsbad, Calif., USA), dissolved in PBS at $50 \mathrm{mg} / \mathrm{ml}$, into the paraflocculus. In 3 cases the paraflocculus alone was injected and in 2 other cases the paraflocculus injection was combined with a 5\% fluorogold (FG; Fluorochrome LLC) injection into the cerebellar vermis and paravermis (the regions outside of the paraflocculus). When combined injections were performed, particular care was taken to avoid the inadvertent labeling of the paraflocculus with FG (fig. 1b).

Experiment 3. To evaluate the percentage of neurons in the arcuate nucleus projecting to the cerebellum, NeuN-specific immunohistochemistry was applied in combination with retrograde tracer injections into the paraflocculus (fig. $1 c ; n=3$ ). In these experiments FG was the preferred tracer as it was more effective than AFD in the measurement of the number of labeled neurons in a particular neuron group.

\section{Postsurgery Tissue Preparation}

In the HRP injection cases, after a survival period of $24 \mathrm{~h}$, mice were deeply anaesthetized with a lethal dosage of pentobarbitone sodium. They were perfused transcardially, sequentially, with heparin saline (40 IU/50 ml saline, $37^{\circ} \mathrm{C}$ ), $4 \%$ paraformaldehyde $\left(4^{\circ} \mathrm{C}, \mathrm{pH} 7.4\right)$ and $10 \%$ sucrose $\left(4^{\circ} \mathrm{C}\right)$. The perfused brains were kept in the same fixative for a further $2 \mathrm{~h}$ and then transferred to $30 \%$ sucrose buffer solution at $4^{\circ} \mathrm{C}$ for $12 \mathrm{~h}$. Serial sections were cut at $40 \mu \mathrm{m}$ on a freezing microtome, and then mounted on gelatine-coated slides prior to the reaction with $3,3^{\prime}, 5,5^{\prime}$ tetramethylbenzidine (TMB) [Mesulam, 1978].

After the retrograde tracer injections, the mice were anaesthetized and perfused on the 14th day postsurgery (FG/AFD or AFD alone) or on the 5th day postsurgery (FG alone), respectively. The brains were cut with a cryostat at a $40-\mu \mathrm{m}$ thickness, mounted on slides and examined under a fluorescence microscope.

For the development study, 2 mice were deeply anaesthetized and perfused at each of four time points (E14, E15, P1 and P5). After postfixation in the same fixative, the brains were cytoprotected in $30 \%$ sucrose, sectioned with freezing microtome and stained with cresyl violet.

\section{Mouse Brain Histology}

We compared the cytological and immunohistochemical characteristics of arcuate neurons with those of the neurons of the inferior olive using cresyl violet staining, acetylcholinesterase (AChE) histochemistry and calbindin-specific immunolabeling employed in mouse brains [adult mice $\mathrm{n}=7$; young mice $(\mathrm{E} 14-\mathrm{P} 5)$ $\mathrm{n}=8$ ]. These techniques were selected on the basis of data from previous published studies of the mouse brain [Franklin and Paxinos, 2008; Watson and Paxinos, 2010]. To perform immunolabeling, sections were pretreated with $1 \% \mathrm{H}_{2} \mathrm{O}_{2}$ in $50 \%$ ethanol to block endogenous peroxidase activity, followed by preincubation in $10 \%$ goat serum. The sections were then incubated with an anticalbindin antibody (C-9848, Sigma) for 2 days at $4^{\circ} \mathrm{C}$, followed by incubation in biotinylated goat anti-mouse IgG and ExtrAvidin peroxidase (Sigma), each for $2 \mathrm{~h}$ at room temperature. The peroxidase complex was visualized by incubating the sections in $0.1 \mathrm{M} \mathrm{PB}$ containing $0.05 \%$ 3,3'-diaminobenzidine (DAB), $0.1 \% \mathrm{H}_{2} \mathrm{O}_{2}$ and $0.04 \%$ nickel ammonium sulphate. Sections were washed, mounted on gelatine-coated slides, dehydrated, cleared and coverslipped using DPX mounting medium (Fluka Chemie AG).

To measure the proportion of precerebellar projecting neurons in the arcuate nucleus, immunofluorescence labeling was employed in brain sections containing the arcuate nucleus and harvested from experiment 3. The sections were blocked in 5\% serum, incubated with anti-NeuN antibody (MAB377, Chemicon) for 2 days at $4^{\circ} \mathrm{C}$, then incubated with Alexa Fluor ${ }^{\circledR} 594$ goat anti-mouse antibody (A11032, Invitrogen) for $2 \mathrm{~h}$ at room temperature. At the end of the incubation, the sections were washed, mounted and coverslipped with mounting medium (Dako, Campbellfield, Vic., Australia).

\section{Antibody Characterization}

Two antibodies were employed in this study: the monoclonal anticalbindin-D-28K recognizing the calbindin-D-28K (also termed vitamin $\mathrm{D}$-dependent calcium-binding protein, or cholecalcin), and the monoclonal anti-NeuN (clone A60) recognizing the DNA-binding, neuron-specific protein NeuN. The specificity of these two antibodies in the tissues was determined by comparison with previously published localization studies [Paxinos et al., 2009; Watson and Paxinos, 2010] and by reacting the tissue with normal serum in place of the specific primary antibodies. No positive reaction was observed in any sections in which the primary antibodies were not included.

\section{Image Analysis and Mapping}

Histological preparations (HRP, Nissl, AChE, and single immunolabeling) were automatically photographed using a Scan- 
Scope digital slide scanner (Model XT, Aperio Technologies Inc., USA). The images were analyzed and extracted with the Aperio ImageScope software, collated with Adobe Photoshop CS4 and mapped with the assistance of an atlas of the mouse brain [Franklin and Paxinos, 2008]. The neurons of the dorsal and ventral tiers of the inferior olive principal nucleus (IOPr), the inferior olive medial nucleus (IOM), and arcuate nucleus medial (ArM) and lateral (ArL) groups were measured in cresyl violet preparations using the Axiovision analysis software (Zeiss, Germany). Neurons with a visible nucleolus within each region of interest $(\mathrm{n}=$ 26-53) were randomly selected for the measurement of the largest diameter of the neuronal soma. The results are presented as mean \pm SEM. Statistical differences were assessed using the $t$ test. To compensate for the error introduced by multiple comparisons, the Bonferroni compensation was employed. After the adjustments of the individual $p$ values, the level of significance was set to $\mathrm{p}<0.05$.

Histological preparations with neuronal tracing combined with immunofluorescence (FG tracing combined with NeuN immunolabeling sections, AFD-tracing combined with calbindinD-28K immunolabeling sections) and single AFD-tracing sections were image captured with a fluorescence microscope (Olympus, BX51) and an attached camera (AxioCam HRc, Zeiss). The delineations for this work were guided by the relevant rat and mouse brain atlases [Paxinos et al., 2007; Franklin and Paxinos, 2008]. The percentages of labeled neurons in the arcuate nucleus were determined in the selected section planes, in which the lateral and/or medial clusters of the arcuate nucleus were contained.

\section{Human Brain Histology}

We examined the arcuate nucleus in five sets of human brainstem sections with no evidence of histopathology. The first of these is a high-quality archive section set that was donated to the University of New South Wales in 1975. The section set contains a one in three series of sections that were cut at $30 \mu \mathrm{m}$ and stained with the Weigert-Pal method to demonstrate myelin. The remaining four sets of normal human medulla sections stained with cresyl violet were obtained from Sydney Brain Bank, courtesy of Professor Glenda Halliday of Neuroscience Research Australia.

\section{Results}

\section{The Histology of the Murine Inferior Olive and} Arcuate Nucleus

We found clusters of neurons on the pial surface of the hindbrain pyramid at the level of the principal nucleus of the inferior olive in the C57BL/6J mouse. We named these clusters the arcuate nucleus because of similarities with the human arcuate nucleus. The inferior olive in the adult mouse is $1.6 \mathrm{~mm}$ long from the rostral pole to the caudal pole as pictured in an atlas of the mouse brain [Franklin and Paxinos, 2008]. In contrast, the length of the cell group we identified as the arcuate nucleus was around $0.6 \mathrm{~mm}$ when measured along the same axis, and was only present in sections containing the rostral part of the inferior olive (fig. 2, 3).
The arcuate nucleus showed AChE histochemical reactivity and calbindin-D-28K immunoreactivity, both of which are prominent in subnuclei of the inferior olive. Preparations demonstrating these two markers were ideal for the delineation of both the arcuate nucleus and the inferior olivary subnuclei. Calbindin is particularly useful as a marker in this region since the ventral half of the caudal hindbrain is almost devoid of calbindin-positive cells, except for the inferior olive neurons [Watson and Paxinos, 2010].

Within the arcuate group in the mouse, there were two identifiable neuronal clusters - a lateral and a medial cluster (ArL and ArM). The rostral ArL was identified in coronal sections as a triangle-shaped group separated dorsally from the IOPr by the medial lemniscus and medulla pyramid (fig. 2a, b, 3a, b). At this rostral level, the mouse arcuate nucleus is very small and contains only a few cells in each section. More caudally, the ArL was continuous with the ventrolateral part of the IOPr (fig. $2 c-e$, $3 \mathrm{c}-\mathrm{e})$.

The ArM appeared to be a medial extension of the ventral IOPr cell streams, and was closely applied to the medial lemniscus on the mediodorsal side (fig. $2 c-e, 3 c-e$ ). In the coronal planes where ArM was present, the IOM was always located dorsal to it. The arcuate nucleus was not present caudal to the IOM (fig. 2f, g, 3f).

In cresyl violet preparations, ArM neurons could be distinguished from those of the IOM neurons by the presence of a very dark Nissl substance in the ArM neuron; this appearance is similar to neurons in the ventral tier of the IOPr. Cell streams connecting the ArM to the ventral tier of the IOPr were also present (fig. $2 c-e, 3 c-e$ ). In AChE preparations, strong labeling was found in the ventral tier of the IOPr, ArL and ArM, but only weak intensity reaction was found in the dorsal tier of the IOPr and the IOM (fig. 3c-f). This supports the idea that the arcuate nucleus is an extension of the IOPr ventral tier.

\section{The Neurons of the Mouse Arcuate Nucleus}

The neurons situated in either the ArL (average diameter: $12.52 \pm 2.11 \mu \mathrm{m}, \mathrm{n}=51)$ or $\operatorname{ArM}(13.88 \pm 2.26 \mu \mathrm{m}$, $\mathrm{n}=41)$ were markedly smaller than those of the dorsal tier of the IOPr $(18.70 \pm 3.31 \mu \mathrm{m}, \mathrm{n}=26)$ and the IOM $(20.14 \pm 2.56 \mu \mathrm{m}, \mathrm{n}=53)$, but very similar to those of the ventral tier of the IOPr $(14.02 \pm 4.02 \mu \mathrm{m}, \mathrm{n}=37)$. Although ArL neurons varied in shape, they commonly had long fusiform cell bodies containing very dark Nissl granules (fig. 4a). Small round neurons were also seen. In contrast, the majority of the neurons in the ArM were small and round, similar to those in the ventral tier IOPr 


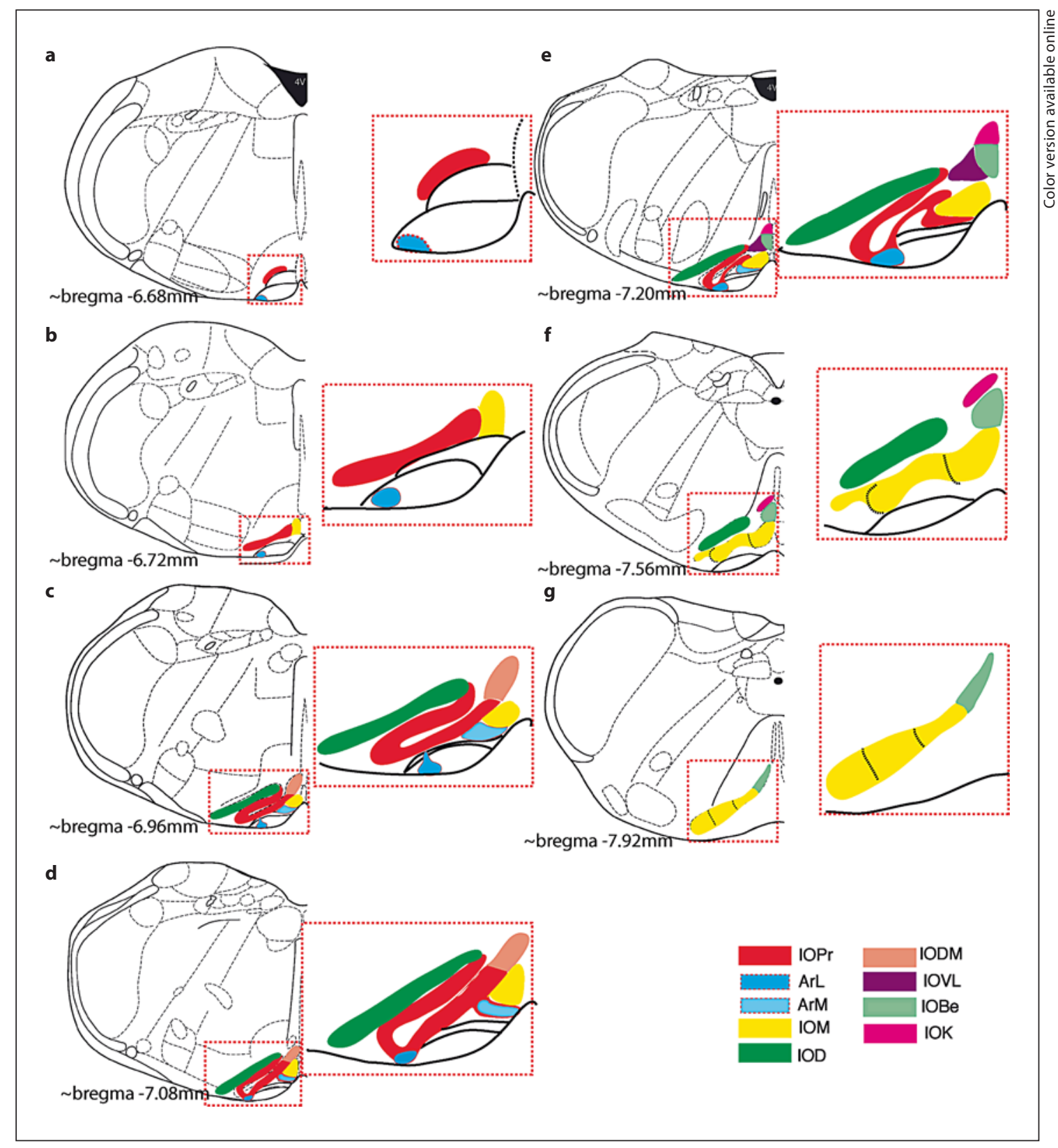

Fig. 2. A diagram of the inferior olive and arcuate nucleus of the C57BL/6J mouse. These diagrams show the location of the inferior olive in a series of hindbrain coronal sections from rostral to caudal (a-g). To the right of each low power diagram is an enlarged schematic drawing to show the subregions of the inferior olive and arcuate nucleus using a color code.

(fig. 4a, b). The neurons in the dorsal tier of the IOPr and IOM were much larger, with the majority being either long, fusiform (dorsal tier of IOPr) or round (IOM) (fig. 4a, b). The major difference between dorsal and ventral IOPr neurons was that the latter contained neurons with very dark Nissl granules making them readily dis- tinguishable from those of the dorsal tier IOPr (fig. 3d, e). The similarity between the histological appearance of the neurons of the arcuate nucleus and the ventral tier of the IOPr further supports the notion that these two structures are anatomically continuous. 

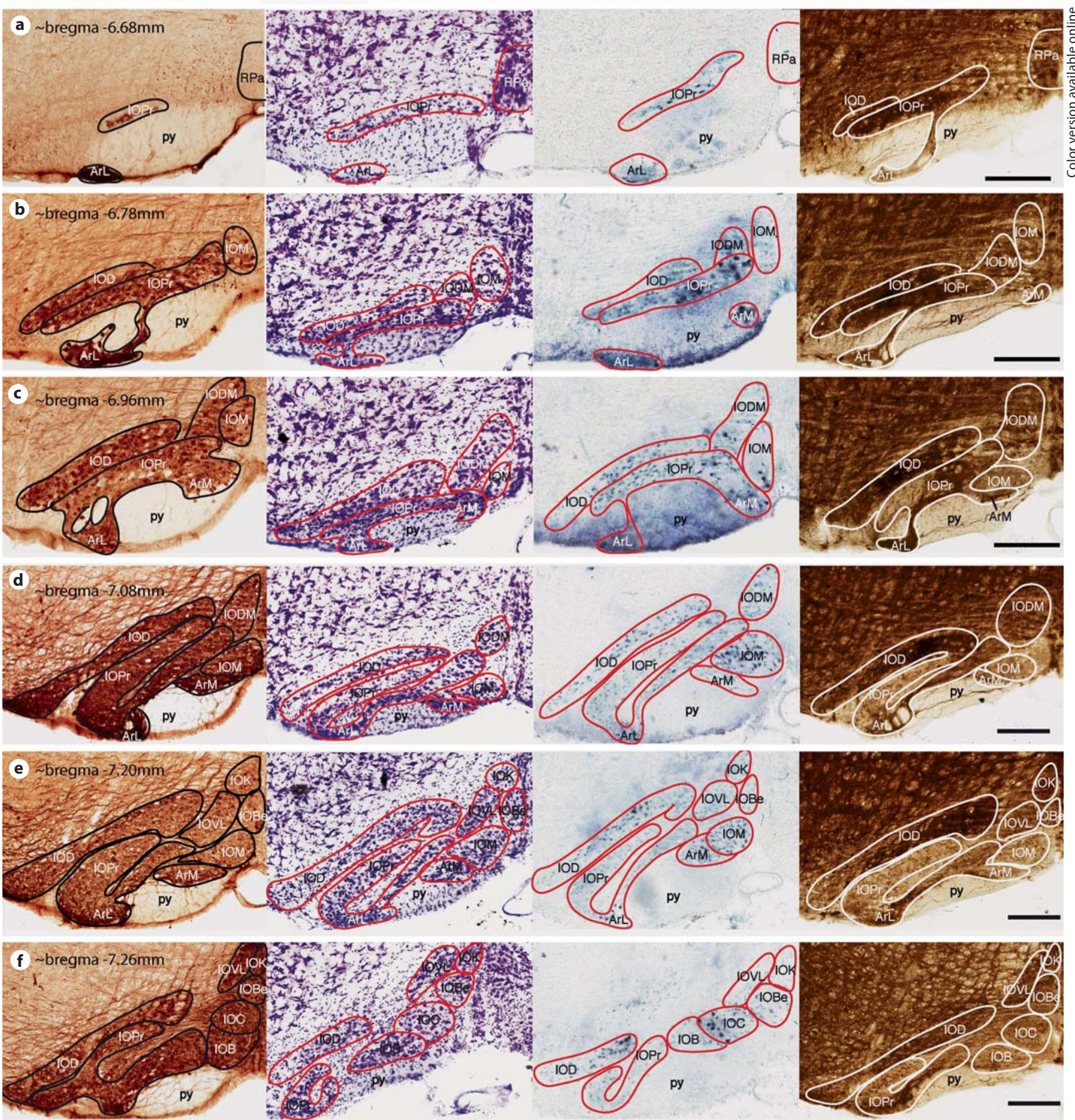

Fig. 3. The topography of the arcuate nucleus in mouse hindbrain sections. A series of coronal sections of the mouse arcuate nucleus from rostral to caudal (a-f). Preparations showing calbindin-D28K-specific labeling (visualized by DAB), Nissl stain (cresyl violet), HRP tracing (color developed by tetramethylbenzidine), and AChE-specific labeling are arranged in the columns from left to right. a-f Scale bar $=200 \mu \mathrm{m}$. 
Other Biological Markers Expressed in the Mouse

Arcuate Nucleus

We found that the neurons of the mouse arcuate nucleus express certain biological markers which were also expressed in inferior olive neurons, but which are not expressed in the major mossy precerebellar nuclei (lateral reticular nucleus, pontine nuclei, external cuneate nucleus and reticulotegmental nucleus of the pons). We studied the expression of a number of genes in images of in situ hybridization preparations on the Allen Brain Institute web site at http://mouse.brain-map.org/welcome.do, using the AGEA tool to localize the expression in the inferior olive and arcuate nucleus. The inferior olive neuron markers we examined were: transmembrane protein 16B; TIGR MGI TC1568100; Src homolog 3 and cysteine rich domain; 5-hydroxytryptamine (serotonin) receptor 5B; Brn3a (POU domain, class 4, transcription factor 1), and RIKEN cDNA 1810041L15 gene. Since the arcuate nucleus expresses all of these genes that characterize inferior olivary neurons (issuing climbing fibers to the cerebellum), we think it is likely that the cerebellar projections of arcuate neurons also form climbing fibers.

\section{Retrograde Labeling after Tracer Injections into the}

\section{Cerebellum in the Mouse}

We examined the cerebellar projection of the arcuate nucleus in two stages; we first injected large areas of cerebellar cortex (fig. 1a - experiment 1), and then made injections aimed at the paraflocculus alone (fig. $1 \mathrm{~b}-$ experiment 2). The paraflocculus injections were carried out in order to investigate a relationship between this region and the arcuate nucleus in other mammals, which has been suggested in the literature [Brodal, 1981; Nagao et al., 1997]. A further injection experiment was designed to measure the percentage of neurons in the arcuate nucleus that project to the cerebellum (fig. 1c - experiment 3).

After a large series of HRP injections into one side of the cerebellar cortex (fig. 1a), tetramethylbenzidine-labeled cells were easily identifiable in the arcuate nucleus and inferior olive. Neurons with blue granular inclusions in the cytoplasm were identified as precerebellar nuclei. HRP-labeled neurons were found in both the rostral and caudal parts of the ArL and ArM (fig. 3, HRP column), confirming that the arcuate nucleus is a precerebellar nucleus.

On the basis of the histological similarity between the neurons of the arcuate nucleus and ventral IOPr and on the continuity of the neurons between these two neighboring structures (fig. $2 c-e, 3 b-e$ ), we regard the mouse
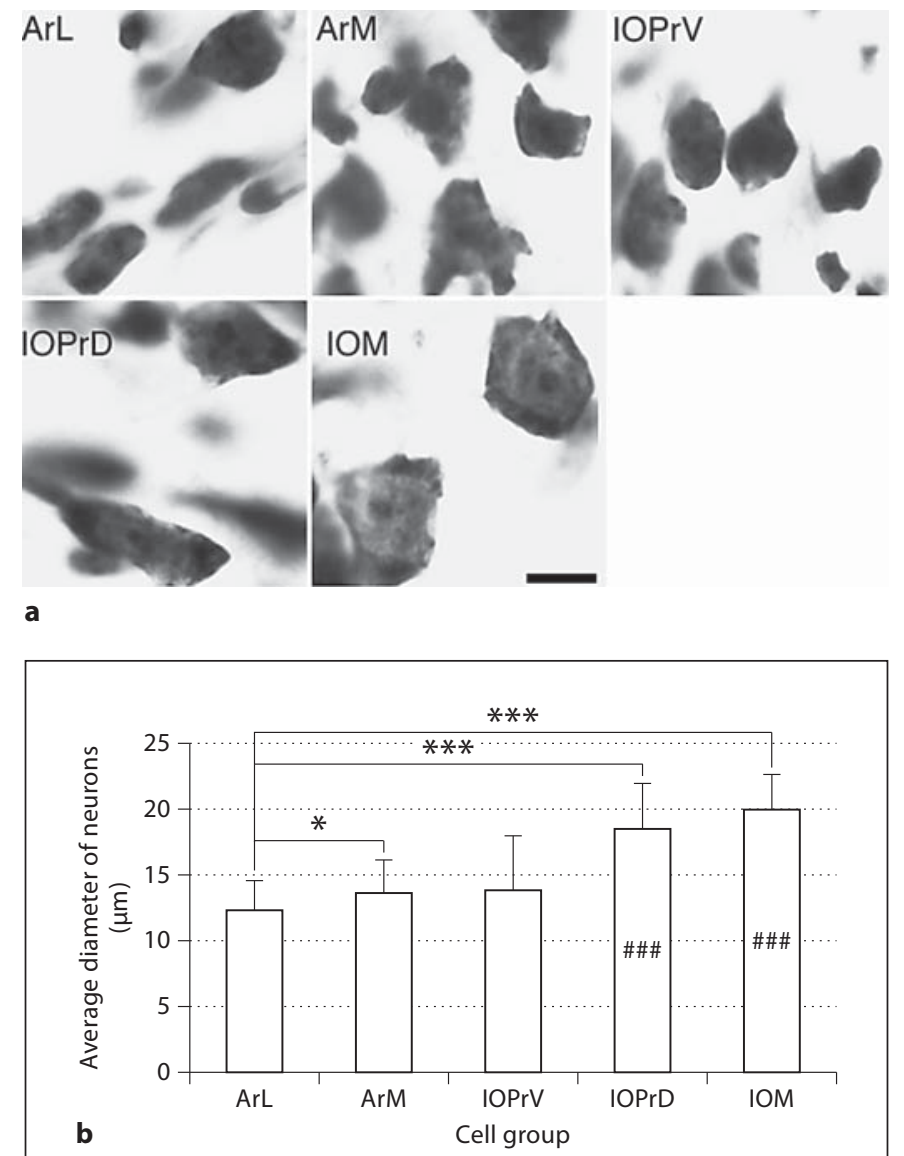

Fig. 4. Morphology of arcuate nucleus neurons. a High-magnification images demonstrating the morphology of the neurons of the arcuate nucleus and neighboring structures (IOPr and IOM). Scale bar $=10 \mu \mathrm{m}$. b Average diameters of neurons in these cell clusters (mean \pm SEM). The significant differences in neuronal diameter between ArL and the other cell groups (ArM, IOPrD, and IOM) are marked as ${ }^{*} \mathrm{p}<0.05$ and ${ }^{* * *} \mathrm{p}<0.001$. The significant differences between ArM and the other cell groups (IOPrD and IOM) are marked as ${ }^{\# \#} \mathrm{p}<0.001$.

arcuate nucleus as an extension of the ventral tier of the IOPr. Studies on the rabbit inferior olive, summarized in Brodal [1981], indicated that the neurons in the ventrolateral IOPr project to the paraflocculus. To find out whether this projection is also a characteristic of the arcuate nucleus, AFD was injected into the paraflocculus, either alone $(n=3)$ or in combination with FG application to the cerebellar vermal and paravermal areas excluding the paraflocculus $(n=2)$. In the cases of vermal and paravermal injections, there were no FG-labeled neurons visible in either the ArL or ArM, showing that the cerebellar ver- 


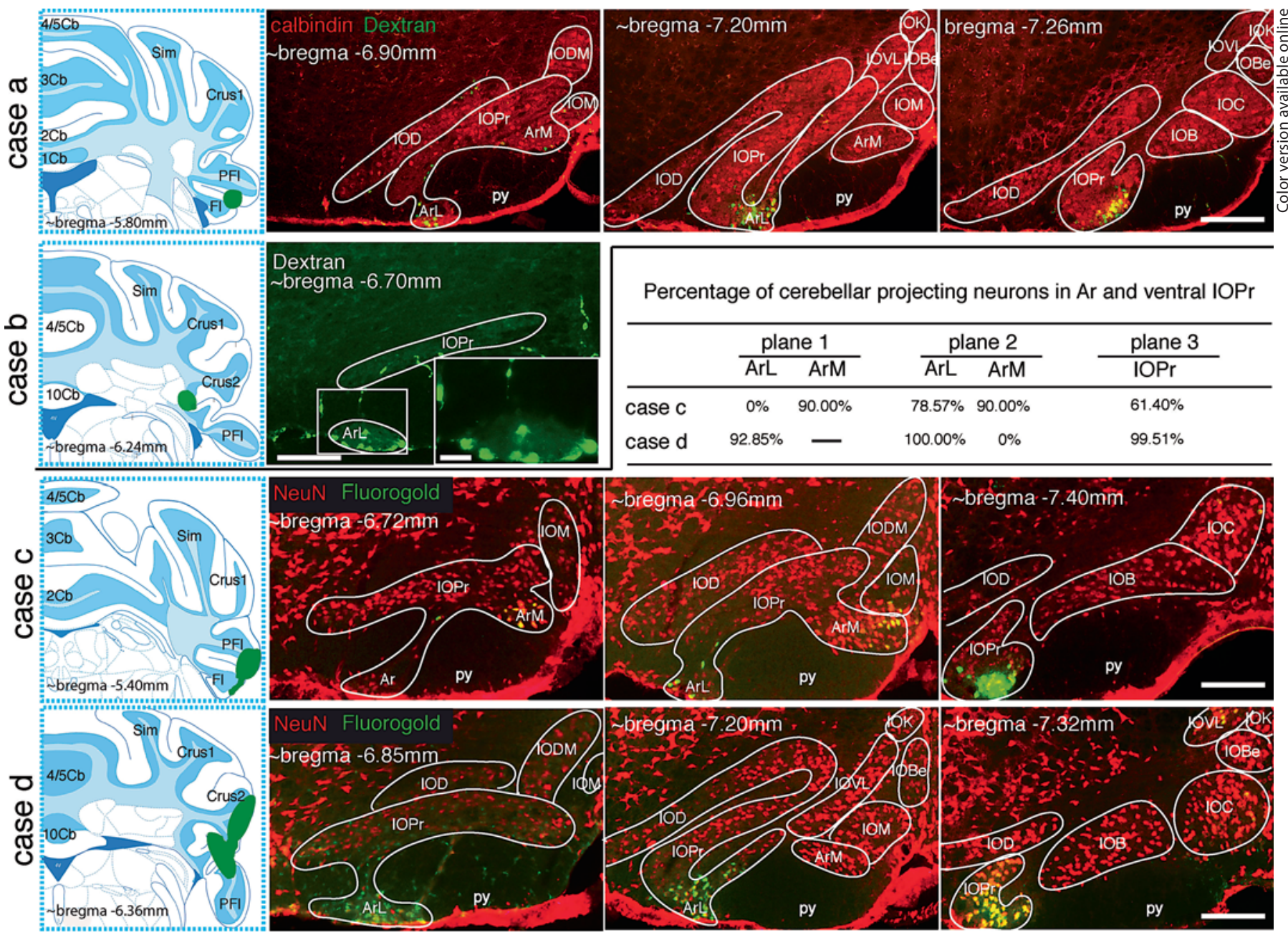

Fig. 5. Results of retrograde tracing from cerebellar injections. Calbindin-D28K-specific immunolabeling (red) prepared from a mouse with AFD injection into the cerebellum. AFD-labeled neurons (green) situated in the arcuate nucleus are arranged along the rostrocaudal axis (case a). AFD-labeled neurons are seen in the most rostral portion of the arcuate nucleus, with a high-power image of the labeled neurons presented in the bottom right corner (case b). Sections demonstrating NeuN-specific immunoreactivity (red) prepared from mice with FG cerebellar injections (cases c and d); the labeled neurons (green) in the arcuate nucleus are arranged along the rostrocaudal axis. The inserted table presents the proportion of arcuate nucleus neurons (at the planes presented in cases $\mathrm{c}$ and d) projecting to the cerebellum. The far left column shows the cerebellar injection sites (areas marked in green) on a series of schematic drawings taken from a mouse brain atlas [Franklin and Paxinos, 2008]. Scale bar $=200 \mu \mathrm{m}$ (cases a-d); 50 $\mu \mathrm{m}$ (high-power image inset case b). mis and the medial portion of the lobules did not receive an arcuate projection. On the other hand, AFD-labeled neurons were found in both the ArL and ArM after all the paraflocculus injections, with the only exception being the most rostral part of the ArL, where no labeled neurons were seen (fig. 5, case a). In contrast, in another case (named as case b in fig. 5) with a single AFD injection into the junction of the paraflocculus and Crus2, AFD-labeled neurons were also found in the most rostral portion of the ArL (fig. 5, case b).
In experiment 3, in which the FG injection was centered in the rostral paraflocculus with slight leakage to the flocculus (fig. 5, case c), cerebellar projecting neurons were found in both the ArL and ArM, but most numerous in the caudal portion of the arcuate nucleus (fig. 5, inserted table). In case d, with an FG injection centered at the dorsal part of the caudal paraflocculus and ventral Crus2, many cerebellar projecting neurons were found in the ArL (fig. 5, inserted table), and only a few neurons were seen in the ArM. 



Fig. 6. The development of the mouse arcuate nucleus at E15 and P1. Photomicrographs showing representative, Nissl-stained sections prepared from mice at E15 (a-d) and P1 (e-h). High-power images of the regions indicated by red squares are presented in the top right corner of the individual images.

The Development of the Mouse Arcuate Nucleus

No inferior olivary subnuclei were visible at E14; the region where the inferior olive would later be formed was occupied by a few cellular clumps, which did not have the appearance of early olivary neurons. Arcuate nucleus neurons were first identified at E15 (fig. 6a, b), but other subgroups of the inferior olive were not clearly visible at this stage, with the inferior olive of each side being represented instead by a single large cluster of neurons in the caudal hindbrain (fig. $6 \mathrm{a}-\mathrm{d}$ ). The cells in the arcuate nucleus formed a stream of neurons in the region where the corticospinal tract and medial lemniscus are formed at later stages of the development. In rats, the extension of the corticospinal tract was found to reach the caudal pole of the medulla at about the time of birth (E21 and P0) [de Kort et al., 1985]. In mice, the leading corticospinal tract axons have reached the 8th cervical segment at P2 [Gianino et al., 1999]. In our study of the brains of P1 mice there was a small bulge on the surface of the hindbrain (fig. $6 \mathrm{e}-\mathrm{h}$ ) where the pyramid would later be identified. Due to the lack of evidence that corticospinal tract fibers have reached the pyramidal decussation at P1 or earlier, we have coined the name pyramidal eminence (pye) to describe this formation at P1 in the mouse.

The arcuate group in the embryo was continuous with the main body of the ventral part of the inferior olive. The cells connecting the inferior olive to the arcuate nucleus were very small and elongated (fig. 6a, high power view), rather similar to those neurons that were observed in the adult mouse arcuate nucleus. At P1, a distinct arcuate nu- cleus was seen and the characteristic subgroups of the inferior olive were distinct and separate (fig. 6e-h), similar to that observed in the arcuate nucleus and inferior olive of the adult mouse. These anatomical similarities were also observed in the $\mathrm{P} 5$ arcuate nucleus and inferior olive.

\section{The Human Arcuate Nucleus}

We found the human arcuate nucleus to be very large, extending along almost the entire rostrocaudal length of the inferior olive. Inspection of our own sections and examination of published illustrations showed that the human arcuate nucleus contains medial and lateral parts, referred to in the literature as the mediodorsal and lateroventral parts [Filiano et al., 1990; Paxinos and Huang, 1995]. According to this scheme, the arcuate nucleus contains two subgroups of neurons, the mediodorsal extension rostrally (fig. $7 \mathrm{a}, \mathrm{d}$ ) and the lateroventral extension caudally (fig. 7c, e, f). Rostrally, the medial extension of the arcuate nucleus formed a thin sheet of cells spreading along the medial and ventral edges of the hindbrain pyramid, extending medially all the way up to the raphe pallidus nucleus. In between the medial lemniscus and the pyramid, the arcuate nucleus formed a spur-like structure pointing to the inferior olive (fig. 7a, d). Occasionally, a small group of arcuate nucleus neurons was seen to be embedded in the pyramid (fig. $7 b, d$ ) in these rostral planes. Although these embedded groups of arcuate neurons are rarely mentioned in the literature [Filiano et al., 1990; Kinney et al., 2007], it can be seen in one of the 

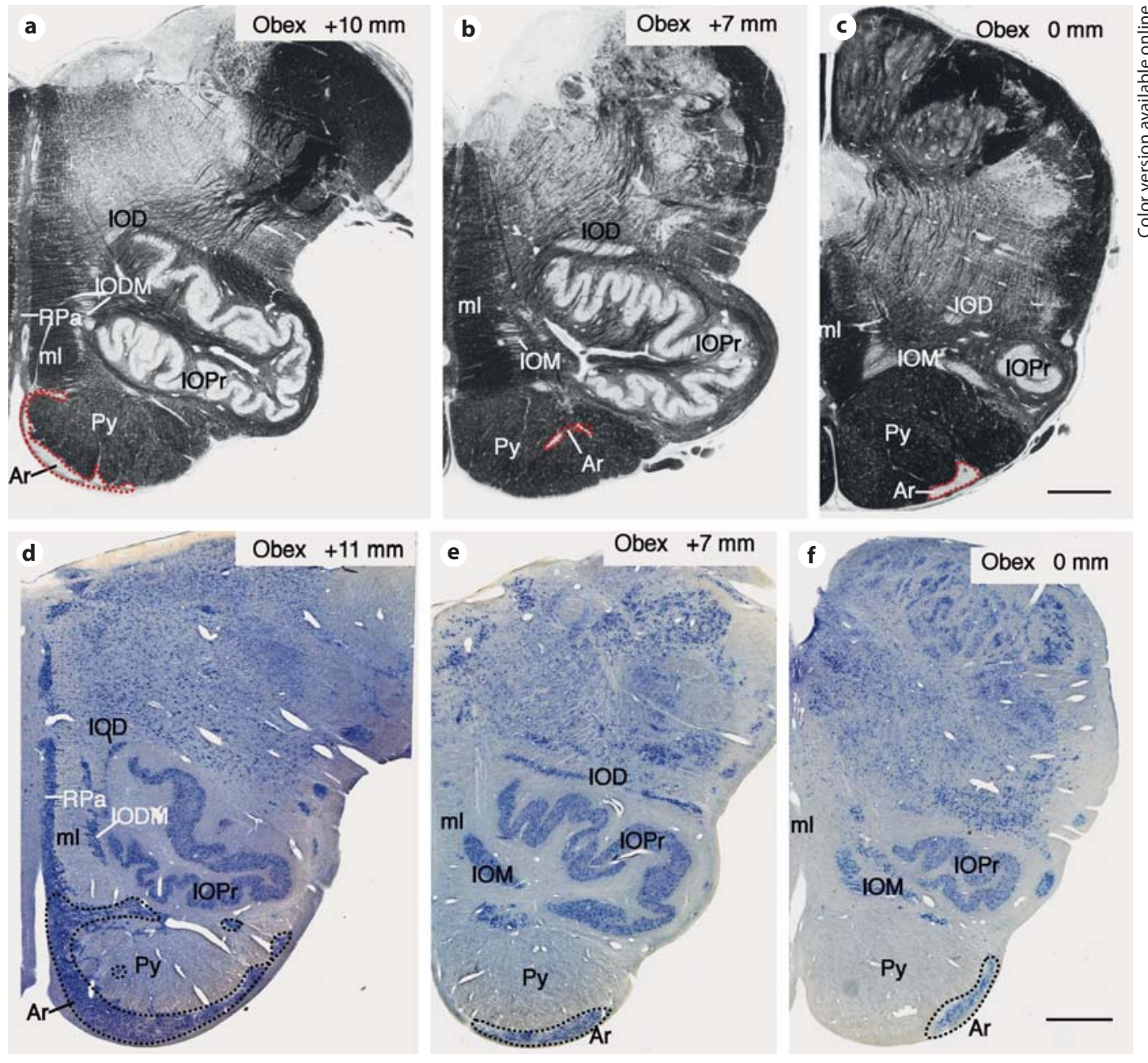

Fig. 7. The human arcuate nucleus. The human arcuate nuclei are shown in coronal sections from rostral to caudal (a-f). Three rostral to caudal sections of the human inferior olive and arcuate nuclei stained with the Weigert-Pal method (a-c), and three rostral to caudal sections of the human inferior olive and arcuate nuclei stained with cresyl violet (d-f). a-f Scale bar $=2 \mathrm{~mm}$.

plates of human medulla in a human brainstem atlas [Paxinos and Huang, 1995]. The extreme caudal part of the lateral extension of the arcuate nucleus was either triangle-shaped or formed a narrow band, but it was still located on the ventral surface of the pyramid (fig. $7 c, f$ ).

\section{The Inconsistent Appearance of the Arcuate Nucleus in Mammals}

The arcuate nucleus was clearly present in all of the C57BL/6J mouse brain samples that we studied. To assess the consistency of this nucleus in other rodents, we looked for the presence of an arcuate nucleus in other strains of mice. We examined calbindin expression in a number of strains of mouse in images on the Allen Brain Institute website (available at http://mousediversity.alleninstitute. org). We found that in mouse strains other than C57BL/6J, the arcuate nucleus is either absent or variably present (see details in table 1).

We also examined our own sets of consecutive sections from Wistar rats, and studied the images of sections of Wistar rats in published atlases [Paxinos and Watson, 2007; Paxinos et al., 2009], but we were not able to identify an arcuate nucleus. In addition, we looked for the presence of an arcuate nucleus in two nonhuman primates (marmoset and rhesus monkey) from recently published atlases [Paxinos et al., 2011, 2012], but we could not identify a cell group representing the arcuate nucleus. We have not found reference to the presence of the arcuate 
nucleus in cats and it is not present in the illustrated sections of the cat brainstem atlases [Snider and Niemer, 1961; Berman, 1968]. We acknowledge that an illustrated series of atlas sections may possibly miss the level where an arcuate nucleus might be present, but the substantial rostrocaudal extent of the nucleus in the mouse and human make this unlikely.

\section{Discussion}

\section{The Rodent Arcuate Nucleus Is Not a Homolog of the Human Arcuate Nucleus}

The human arcuate nucleus is located in the hindbrain, on the ventral aspect of the pyramid. It contains two subgroups of neurons, the mediodorsal extension at the rostral arcuate nucleus and the lateroventral extension at the caudal arcuate nucleus. The mouse arcuate nucleus is similarly positioned and also contains two major clusters, named ArL and ArM in this study. We thought at first that the mouse arcuate nucleus must be a homolog of the human arcuate nucleus, but we were mistaken. The mouse arcuate nucleus is calbindin positive, like the inferior olive, but the human arcuate nucleus is not [Joan Bazier, pers. comm., 2011]. The human arcuate nucleus appears to be a caudal homolog of the pontine nuclei, as originally suggested by Essick [1912]. This presence of a large arcuate nucleus in humans may correlate with the massive expansion of the pontocerebellar system in the human brain. It may be related to the presence of additional 'pontine' nuclei (in the form of the gamma nuclei) in the human hindbrain [Paxinos and Huang, 1995].

In searching for common features, we compared the presence of a number of molecular markers in the mouse arcuate with the human arcuate nucleus. We found that both mouse and human nuclei express VGLUT2 (Slc17a6) and Tph2, but this commonality seems to be much less important than the absence of calbindin in the human arcuate nucleus.

\section{The Mouse Arcuate Nucleus Is a Precerebellar \\ Nucleus}

As noted above, the human arcuate nucleus has been traditionally considered to be a precerebellar nucleus [Zec et al., 1997]. In our study, we confirmed that the mouse arcuate nucleus projects to the paraflocculus of the contralateral cerebellar cortex. Some arcuate efferents may also terminate in the contralateral flocculus and the lateral portion of the cerebellar hemisphere, especially the Crus2, but these projections remain to be con-
Table 1. The presence or absence of the hindbrain arcuate nucleus in the brains of adult mice belonging to seven different mouse strains

\begin{tabular}{llll}
\hline Strain & Gender & Brains, $n$ & Arcuate nucleus present, $\mathrm{n}$ \\
\hline 129S1/Svlmj & male & 4 & 3 \\
C57BL/6J & male & 4 & 4 \\
CAST/EIJ & male & 4 & 0 \\
DBA/2J & male & 4 & 0 \\
PWD/PhJ & male & 2 & 0 \\
SPRET/EIJ & male & 4 & 0 \\
WSB/EIJ & male & 2 & 0 \\
\hline
\end{tabular}

The brains were prepared to show calbindin-28k expression. The images were studied on the website of the Allen Brain Institute for Brain Science.

firmed. The exclusively contralateral projection character of the arcuate nucleus is the same as that of the inferior olive. In this respect, the inferior olive and the arcuate nucleus differ from the precerebellar nuclei that give rise to mossy fibers because these nuclei project either bilaterally or ipsilaterally to the cerebellum [Fu et al., 2011]. Moreover, the arcuate nucleus was found to express several biomarkers that were also expressed in the inferior olive, and which were not expressed in the mossy precerebellar nuclei (lateral reticular nucleus, pontine nuclei, external cuneate nucleus and reticulotegmental nucleus of the pons).

The inferior olive, arcuate nucleus and Purkinje cell layers are the three major calbindin-positive groups located in the hindbrain [Watson and Paxinos, 2010]. Calbindin is a fast $\mathrm{Ca}^{2+}$ protein and has been reported to be involved in the firing regulation of the Purkinje cells [Servais et al., 2005]. It has been reported that calbindin is a reliable marker for the whole olivocerebellar projection in adult rodents [Scotti, 1995]. It is therefore reasonable to consider the arcuate nucleus a member of this climbing fiber precerebellar system.

\section{The Mouse Arcuate Nucleus and the Inferior Olive}

Both Arise in the Rhombic Lip

In their landmark histogenetic studies of the rhombic lip, both Essick [1912] and Harkmark [1954] observed that both the inferior olive and the pontine nuclei arose from the rhombic lip, which has been confirmed by recent fate mapping studies such as that of Rodriguez and Dymecki [2000]. Essick [1912] studied human embryos and reported on the development and migration of the 
arcuate nucleus. He observed that the arcuate neurons migrated along with the olivary neurons ('the olivo-arcuate migration'), and this finding seems to be consistent with our conclusions. However, Essick also found that the most rostral part of the arcuate nucleus was continuous with the basilar pontine nuclei, and therefore proposed, in the absence of other data, it was likely that the arcuate nucleus was an outlying representative of the pontine gray. However, Bloch-Gallego et al. [2005] found that the arcuate neurons migrated along with the pontine nuclei in the extramural stream, which supports the conclusion drawn by Essick that the arcuate neurons are related to the pontine nuclei. On the other hand, our immunohistochemical and gene marker data definitely show that the mouse arcuate nucleus shares markers with olivary neurons and not with pontine neurons.

We have found that the mouse arcuate homolog shares the expression of many gene markers [transmembrane protein 16B, TIGR MGI TC1568100, Src homolog 3 and cysteine rich domain, 5-hydroxytryptamine (serotonin) receptor 5B, Brn3a and RIKEN cDNA 1810041L15 gene] with inferior olivary neurons, and not with pontine neurons. The main biomarker we applied in this work, the calbindin-D-28K, was widely distributed in all the subregions of the inferior olive and arcuate nucleus, but not in the pontine nucleus. In addition, the arcuate homolog cells are in some sections directly continuous with the cells of the inferior limb of the inferior olive (ventral IOPr) in the mouse, express more similar AChE pattern with the ventral IOPr than other subregions of the inferior olive, and are in similar cellular size as those in ventral IOPr. We therefore conclude that the arcuate nucleus is an extension of the ventral IOPr.

Since both the inferior olive and pontine neurons are derived from the rhombic lip in the embryo [Essick, 1912; Harkmark, 1954; Rodriguez and Dymecki, 2000], we consider it likely that the mouse arcuate neurons are also derived from the rhombic lip.

\section{The Relationship of the Arcuate Nucleus to the Serotonergic Raphe}

Because the medial part of the arcuate nucleus lies adjacent to the raphe pallidus serotonergic neurons, some authors have favored the possibility that the arcuate nucleus in humans is a subgroup of the raphe pallidus [Benarroch et al., 2007]. Others have interpreted the presence of 5-HT neurons embedded with the anatomic boundary of the arcuate nucleus as a ventral and ventrolateral extension of the raphe pallidus [Paterson et al., 2006a; Kinney et al., 2007], an observation further supported by the demon- stration of connections between the arcuate nucleus and raphe with DiI tracing in the human fetal medulla [Zec et al., 1997]. On the other hand, we have presented evidence showing that the arcuate nucleus in mice is functionally and anatomically continuous with the IOPr. The arcuate nucleus and IOPr share a number of characteristics: their cytology is very similar; they both project to the paraflocculus of the cerebellum; both share biological markers that are specific to climbing fiber issuing precerebellar neurons, and they both express biological markers expressed in the human arcuate nucleus. We found a small number of Tph2-positive cells in the rostral ArL in images on the website of the Allen Brain Institute. We suggest that a small subpopulation of serotonergic cells invades the main part of the arcuate nucleus at some point.

\section{The Relationship between the Pyramidal Tract and the Developing Arcuate Nucleus}

In the rat, the inferior olive neurons are born at E14 and E15, and are mature at E21 [Ellenberger et al., 1969]. In the mouse, the early development of the inferior olive occurs at E14-E15, followed by its maturation at about E16-birth $(E 19=$ P0) [Goffinet, 1983]. Our observations on the time course of the arcuate nucleus development are consistent with this. Because of the inconsistent appearance of the arcuate nucleus in different strains of mice, we have explored the relationship between the inferior olive development and the formation of the hindbrain pyramid. Sema6A is a major contributor to the guidance of the axons forming the corticospinal tract; low level Sema6A-specific immunoreactivity was detected along the trajectory of growing corticospinal axons in the basilar pons at E17.5 and in the pyramid at P1 [Rünker et al., 2008]. In addition, there were moderate to high levels of expression in most parts of the inferior olive at E17.5 [Rünker et al., 2008]. However, the ventrolateral portion of the IOPr, which we believe to be the site of origin of arcuate nucleus neurons, was the only region of the inferior olive without Sema6A expression. It is possible that this area of the inferior olive allows corticospinal axons to pass through it as they grow caudally, therefore causing the arcuate neurons to be separated from the ventral IOPr. We speculate that differing patterns of early gene expression (like the Sema6A expression in mice), or differences in the timing of expression, might account for the absence of the arcuate nucleus in many mouse strains and the lack of evidence for its existence in rats, monkeys and cats.

Since the arcuate nucleus is a prominent structure in the human and mouse brains, but is not evident in the rat 
or monkey brains we examined, it is reasonable to ask why its presence is inconsistent in mammals. We believe that the equivalent cell group in most mammals is probably the ventral tier of the IOPr. We think it is likely that the minor variations in corticospinal axon growth might serve to separate the arcuate neuron group from the remainder of the ventral tier of the IOPr in some mammals. Based on our studies of the projection of the arcuate nucleus to the paraflocculus, we suggest that any part of the ventral tier of IOPr that projects to the paraflocculus is likely to be a homolog of the mouse arcuate nucleus.

\section{The Functional Role of the Arcuate Nucleus}

Hypoplasia and degeneration of the arcuate nucleus were found in stillborn infants [Zec et al., 1997; Matturri et al., 2002] and in cases of multiple system atrophy [Benarroch et al., 2007], and these facts have led to a suggestion that the human arcuate nucleus plays a role in central chemoreception [Zec et al., 1997]. In the current study, we observed marked changes of the respiratory frequency during and after the stimulation of the paraflocculus, the cerebellar target of the arcuate nucleus (preliminary data not shown). Although the cerebellum is not traditionally considered to be a component of circuits involved in respiratory control, breathing involves cyclic motor activity that requires cerebellar coordination
[Chen et al., 2005]. This suggestion is supported by the finding that cerebellar hypoplasia was associated with respiratory control disorders and mental retardation in newborn human infants [González de Dios et al., 1995]. In addition, disordered respiratory control has been observed in children with partial cerebellar resections [Chen et al., 2005]. We suggest that the arcuate nucleus and its cerebellar target might to some extent be involved in respiratory function.

\section{Acknowledgements}

This project was supported by the Australian Research Council Thinking Systems Initiative (TS0669860). The study was supported by an NHMRC (National Health and Medical Research Council) Australia Fellowship Grant awarded to Dr. George Paxinos (Grant No. 568605). We acknowledge the important contribution from the open online resources of the Allen Brain Institute. We thank the Sydney Brain Bank (which is supported by Neuroscience Research Australia, the University of New South Wales and the National Health and Medical Research Council of Australia) for providing human brainstem sections. We thank Dr. Joan Baizer, University at Buffalo, for her vital advice on calbindin. We greatly appreciate the assistance and support provided by Dr. Emma Schofield, Dr. Zoltán Rusznák and Dr. DeYi Duan. We particularly appreciate the contribution of two anonymous reviewers who corrected errors and gave us many valuable suggestions, which have helped us improve this paper.

\section{References}

Ahlsen G (1981): Retrograde labelling of retinogeniculate neurones in the cat by HRP uptake from the diffuse injection zone. Brain Res 223:374-380.

Benarroch EE (2007): Brainstem respiratory control: substrates of respiratory failure of multiple system atrophy. Mov Disord 22: $155-161$.

- Benarroch EE, Schmeichel AM, Low PA, Parisi JE (2007): Depletion of putative chemosensitive respiratory neurons in the ventral medullary surface in multiple system atrophy. Brain 130:469-475.

Berman AL (1968): The Brain Stem of the Cat: A Cytoarchitectonic Atlas with Stereotaxic Coordinates. Madison, University of Wisconsin Press.

-Bloch-Gallego E, Causeret F, Ezan F, Backer S, Hidalgo-Sanáhez M (2005): Development of precerebellar nuclei: instructive factors and intracellular mediators in neuronal migration, survival and axon pathfinding. Brain Res Brain Res Rev 49:253-266.
Brodal A (1981): Neurological Anatomy in Relation to Clinical Medicine. New York, Oxford University Press.

-Chen ML, Witmans MB, Tablizo MA, Jubran RF, Turkel SB, Tavaré CJ, Keens TG: (2005): Disordered respiratory control in children with partial cerebellar resections. Pediatr Pulmonol 40:88-91.

de Kort EJ, Gribnau AA, van Aanholt HT, Nieuwenhuys R (1985): On the development of the pyramidal tract in the rat. I. The morphology of the growth zone. Anat Embryol (Berl) 172: 195-204.

-Ellenberger C Jr, Hanaway J, Netsky MG (1969): Embryogenesis of the inferior olivary nucleus in the rat: a radioautographic study and a re-evaluation of the rhombic lip. J Comp Neurol 137:71-79.

Essick CR (1912): The development of the nuclei pontis and the nucleus arcuatus in man. Am J Anat 13:25-54.

-Filiano JJ, Choi JC, Kinney HC (1990): Candidate cell populations for respiratory chemosensitive fields in the human infant medulla. J Comp Neurol 293:448-465.
Filiano JJ, Kinney HC (1992): Arcuate nucleus hypoplasia in the sudden infant death syndrome. J Neuropathol Exp Neurol 51:394403.

Folgering H, Kuyper F, Kille JF (1979): Primary alveolar hypoventilation (Ondine's curse syndrome) in an infant without external arcuate nucleus. Case report. Bull Eur Physiopathol Respir 15:659-665.

Folkerth RD, Zanoni S, Andiman SE, Billiards SS (2008): Neuronal cell death in the arcuate nucleus of the medulla oblongata in stillbirth. Int J Dev Neurosci 26:133-140.

Franklin KBJ, Paxinos G (2008): The Mouse Brain in Stereotaxic Coordinates. San Diego, Elsevier Academic Press.

Fu Y, Tvrdik P, Makki N, Paxinos G, Watson C (2011): Precerebellar cell groups in the hindbrain of the mouse defined by retrograde tracing and correlated with cumulative Wnt1-Cre genetic labeling. Cerebellum 10: 570-584. 
Gianino S, Stein SA, Li H, Lu X, Biesiada E, Ulas J, Xu XM (1999): Postnatal growth of corticospinal axons in the spinal cord of developing mice. Brain Res Dev Brain Res 112:189204.

Goffinet AM (1983): The embryonic development of the inferior olivary complex in normal and reeler (rlORL) mutant mice. J Comp Neurol 219:10-24.

-González de Dios J, García-Alix A, Cabañas F, Quero J, Moya M (1995): Cerebellar hypoplasia in the newborn: association with respiratory control disorders and mental retardation. Rev Neurol 23:1041-1046.

Gozal D, Hathout GM, Kirlew KA, Tang H, Woo MS, Zhang J, Lufkin RB, Harper RM (1994): Localization of putative neural respiratory regions in the human by functional magnetic resonance imaging. J Appl Physiol 76: 2076-2083.

Harkmark W (1954): Cell migrations from the rhombic lip to the inferior olive, the nucleus raphe and the pons: a morphological and experimental investigation on chick embryos. J Comp Neurol 100:115-209.

-Kinney HC, Belliveau RA, Trachtenberg FL, Rava LA, Paterson DS (2007): The development of the medullary serotonergic system in early human life. Auton Neurosci 132:81102.

Kinney HC, Filiano JJ, Sleeper LA, Mandell F, Valdes-Dapena M, White WF (1995): Decreased muscarinic receptor binding in the arcuate nucleus in sudden infant death syndrome. Science 269:1446-1450.

-Matturri L, Minoli I, Lavezzi AM, Cappellini A, Ramos S, Rossi L (2002): Hypoplasia of medullary arcuate nucleus in unexpected late fetal death (stillborn infants): a pathologic study. Pediatrics 109:E43.
Mesulam MM (1978): Tetramethyl benzidine for horseradish peroxidase neurohistochemistry: a non-carcinogenic blue reaction product with superior sensitivity for visualizing neural afferents and efferents. J Histochem Cytochem 26:106-117.

Mikhail Y, Ahmed YY (1975): Outline of the arcuate nucleus in the human medulla oblongata. Acta Anat (Basel) 92:285-291.

Nagao S, Kitamura T, Nakamura N, Hiramatsu T, Yamada J (1997): Differences of the primate flocculus and ventral paraflocculus in the mossy and climbing fiber input organization. J Comp Neurol 382:480-498.

Natti E (1991): Central respiratory chemoreceptors; in Haddad G, Farber J (eds): Developmental Neurobiology of Breathing. New York, Dekker, pp 341-372.

Paterson DS, Thompson EG, Kinney HC (2006a): Serotonergic and glutamatergic neurons at the ventral medullary surface of the human infant: observations relevant to central chemosensitivity in early human life. Auton Neurosci 124:112-124.

- Paterson DS, Trachtenberg FL, Thompson EG, Belliveau RA, Beggs AH, et al. (2006b): Multiple serotonergic brainstem abnormalities in sudden infant death syndrome. JAMA 296:2124-2132.

Paxinos G, Halliday G, Watson C, Koutcherov Y, Wang HQ (2007): Atlas of the Developing Mouse Brain at E17.5, P0, and P6. Burlington, Academic Press.

Paxinos G, Huang XF (1995): Atlas of the Human Brainstem: San Diego, Academic Press.

Paxinos G, Petrides M, Watson C (2011): The Rhesus Monkey Brain in Stereotaxic Coordinates. Amsterdam, Elsevier, in press.
Paxinos G, Watson C (2007): The Rat Brain in Stereotaxic Coordinates. London, Academic Press.

Paxinos G, Watson C, Carrive P, Kirkcaldie M, Ashwell KW (2009): Chemoarchitectonic Atlas of the Rat Brain. San Diego, Academic Press.

Paxinos G, Watson C, Petrides M, Rosa M, Tokuno H (2012): The Marmoset Brain in Stereotaxic Coordinates. San Diego, Elsevier Academic Press.

Rodriguez CI, Dymecki SM (2000): Origin of the precerebellar system. Neuron 27:475-486.

-Rünker AE, Little GE, Suto F, Fujisawa H, Mitchell KJ (2008): Semaphorin-6A controls guidance of corticospinal tract axons at multiple choice points. Neural Dev 3:34.

- Scotti AL (1995): Calbindin D28k in the olivocerebellar projection: a light and electron microscope study. J Anat 187(Pt 3):649-659.

-Servais L, Bearzatto B, Schwaller B, Dumont M, De Saedeleer C, et al. (2005): Mono- and dual-frequency fast cerebellar oscillation in mice lacking parvalbumin and/or calbindin D-28k. Eur J Neurosci 22:861-870.

Snider RS, Niemer WT (1961): A Stereotaxic Atlas of the Cat Brain. Chicago, University of Chicago Press.

Tomycz ND, Haynes RL, Schmidt EF, Ackerson K, Kinney HC (2010): Novel neuropathologic findings in the Haddad syndrome. Acta Neuropathol 119:261-269.

Watson C, Paxinos G (2010): Chemoarchitectonic Atlas of the Mouse Brain. San Diego, Academic Press.

Zec N, Filiano JJ, Kinney HC (1997): Anatomic relationships of the human arcuate nucleus of the medulla: a DiI-labeling study. J Neuropathol Exp Neurol 56:509-522. 